\title{
Origin and role of neural signatures in bursting neurons
}

\author{
Roberto Latorre, Francisco B. Rodríguez and Pablo Varona \\ Grupo de Neurocomputación Biológica (GNB). Dpto. de Ingeniería Informática. \\ Escuela Politécnica Superior. Universidad Autónoma de Madrid. 28049 Madrid. Spain.
}

\begin{abstract}
A traditional view in neuroscience is that information arriving through one channel, i.e. a synapse, is encoded through a single code in the signal, e.g., the rate or the precise timing of the incoming events. However, not all the neural readers have to be interested in the same aspect of a common input signal, especially in multifunctional networks that can take advantage of several simultaneous codes. Multiple codes can be used to discriminate or contextualize certain inputs, even in single neurons. Dynamical mechanisms can add to the existing hard-wired connectivity for this task. Recent experiments have revealed the existence of neural signatures in the activity of bursting cells of invertebrate central pattern generators. These signatures consist of cell-specific spike timings in the bursting activity of the neurons. The signatures coexist with the information encoded in the frequency and/or phase relationships of the slow waves. The functional role of these neural fingerprints is still unclear. Based on experiments and using conductance-based models, we discuss the origin and the role of neural signatures as a part of a multicoding strategy for single cells in different types of neural circuits.
\end{abstract}

Keywords: Multicoding - Central Pattern Generators (CPG) - Interspike Interval (ISI) analysis spiking-bursting activity

PACS: 87.19.La, 87.18.Sn, 05.45.Xt

\section{INTRODUCTION}

Neural bursting activity is present in many cells of different nervous systems. Depending on the particular system under study, bursts are interpreted as pathological states [1], as a very reliable mechanism for transmitting information [2], or as an essential means to induce plasticity [3]. Bursts are traditionally considered as unitary events, and thus the temporal distribution of action potentials inside the bursts is often disregarded. Only recently there has been some attention to assess the specific role of the spiking activity in bursting neurons [4, 5, 6, 7] (see Fig. 1).

Bursting activity is very common in Central Pattern Generators (CPGs), relative simple neural networks for the production of motor rhythms. CPGs are multifunctional circuits highly specialized to produce a rhythmic sequence to control a movement that must be repeated in time. CPGs from invertebrates are particularly suitable for experimental purposes, as these networks are easy accessible and all their neurons and connections can be identified. The intraburst spike distribution of CPG neurons has not been analyzed in great detail since, typically, it is thought that the slow wave dynamics is the main factor shaping the rhythmic behavior of the system. However, several recent experimental findings point out that the temporal structure of the bursts can be important for CPG neurons. In particular, the first spikes in the bursting activity of pyloric cells are highly reliable $[8,9,10]$ and they display neural signatures in their intraburst interspike

CP887, Cooperative Behavior in Neural Systems: Ninth Granada Lectures edited by J. Marro, P. L. Garrido, and J. J. Torres

(C) 2007 American Institute of Physics 978-0-7354-0390-1/07/\$23.00 
interval (ISI) distributions [11, 12]. The neural signatures consist of reproducible cellspecific spike timings in the bursting activity of each neuron.

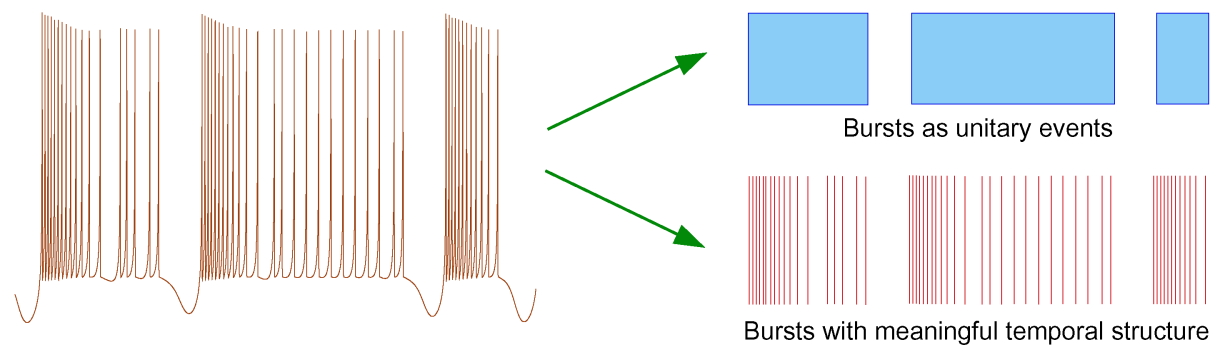

FIGURE 1. (Color online) Traditional and new views of bursting activity.

There are many open questions related to the discovery of the neural signatures. In this paper we review some of our modeling work about the origin of the neural signatures and their role in implementing multiple codes in spiking-bursting activity [13, 14, 15, $16,17]$.

\section{INTRINSIC DYNAMICS AND CONNECTIVITY}

A first open question from the in vitro experiments is related to the origin of the neural signatures. Intrinsic dynamics, network connections, and the effect of external neuromodulation are all factors that could shape the intraburst spike timings. To isolate these factors in in vitro experiments is a hard task. We have used realistic models of CPG neurons and networks to pursue this question.

\section{Single neuron models}

To analyze the dependence of neural signatures on the intrinsic cell properties and on the network architecture we have used Komendantov-Kononenko (KK) model neurons [18]. This conductance-based model has a rich dynamic behavior with the ability to generate the characteristic bifurcations in the spiking-bursting behavior observed in the activity of isolated CPG living cells. As a function of the maximum conductances of the ionic channels, the model can be set into a regular spiking-bursting or into a chaotic spiking-bursting activity. The equations that describe the dynamics of the model and the parameters used to obtain each type of behavior can be found in [18].

Fig. 2 shows the regular and chaotic spiking-bursting activity in a KK single neuron model. The intraburst spike activity is typically represented by interspike interval return maps $[19,20,21,4,5,11]$. These return maps plot $I S I_{i}$ vs. $I S I_{i+1}$ and are a useful tool to characterize the cell-specific intraburst interspike timing distributions. Right panels in Fig. 2 show the neural signatures of regular and chaotic spiking-bursting modes represented by return maps. 

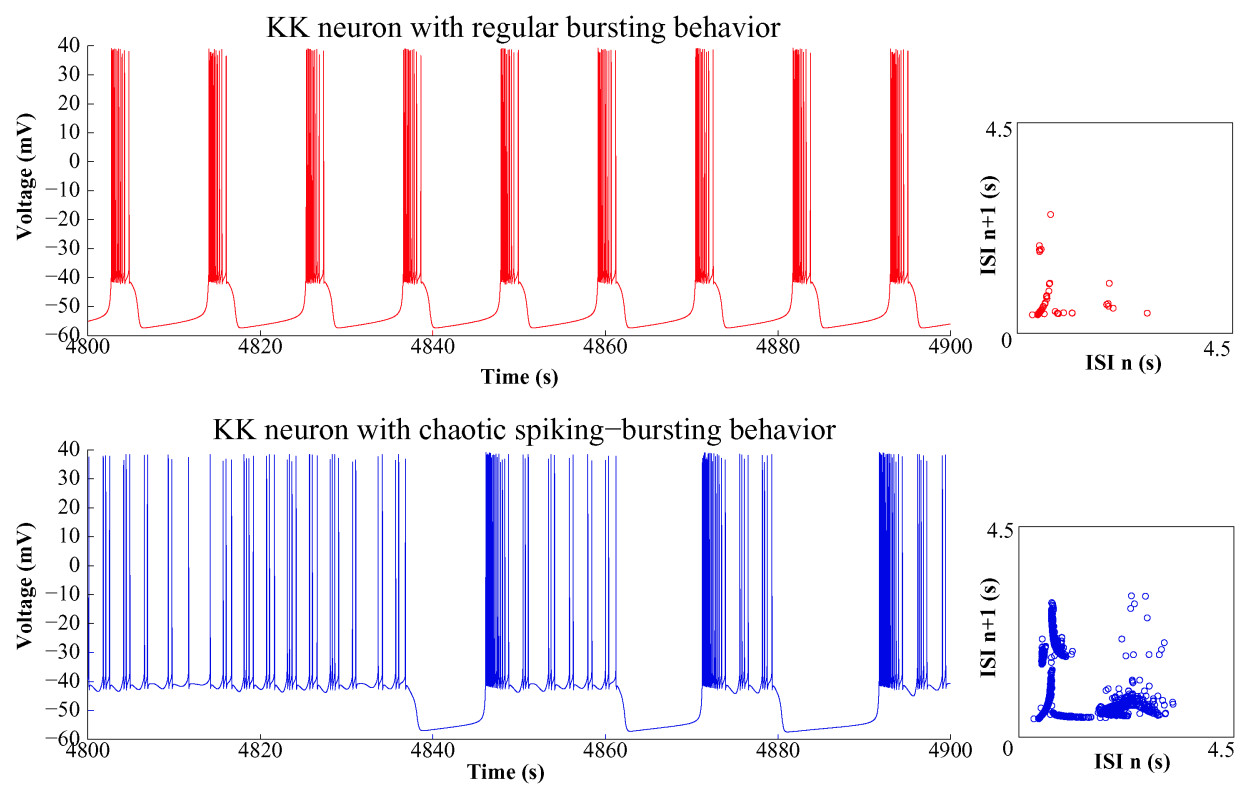

FIGURE 2. (Color online) Different bursting modes of an isolated KK single neuron model. Right panels show the ISI return maps of the intraburst spiking activity.

\section{Network models}

For the network analysis we have used two different topologies depicted in the right panels of Fig. 3. These circuits are inspired in the pyloric CPG of crustacean. Both networks are built out of two subcircuits: the AB-PD1-PD2 neurons, which are mutually connected through electrical coupling (represented with resistors), and the AB-LP-PY neurons that are connected with chemical synapses. There are two types of chemical connections in the first circuit: slow (represented with dotted lines) and fast (represented with solid lines). The second circuit (lower right panel in Fig. 3) does not include slow chemical connections. With these two circuits we will be able to analyze the dependence of the spiking behavior on the architecture of connections. In the AB-PD1-PD2 subnetwork (often called the pacemaker group), the neurons are electrically coupled with symmetric connections [13]. As a consequence, the subthreshold membrane potentials of these neurons are synchronized. Physiologists have always recorded a regular spikingbursting activity in the $\mathrm{AB}$ neuron even when isolated from the rest of the circuit. For this reason, in all our models the $\mathrm{AB}$ model neuron has a regular behavior. Conversely, the isolated PD model neurons are set into a non-regular regime since this is the behavior observed in the experiments on isolated cells.

The AB/PD-LP-PY network comprises the pacemaker group and the LP and PY neurons. The later have connections to and from the pacemaker group by means of chemical synapses. With this architecture of connections, the living pyloric CPG generates a robust triphasic rhythm: there is an alternating regular bursting activity between the pace- 
maker group, the LP and the PY neuron. Both model circuits reproduce this behavior (see Fig. 3). The details of the connection models are reported in [13].

Circuit with Slow Chemical Synapses (A)
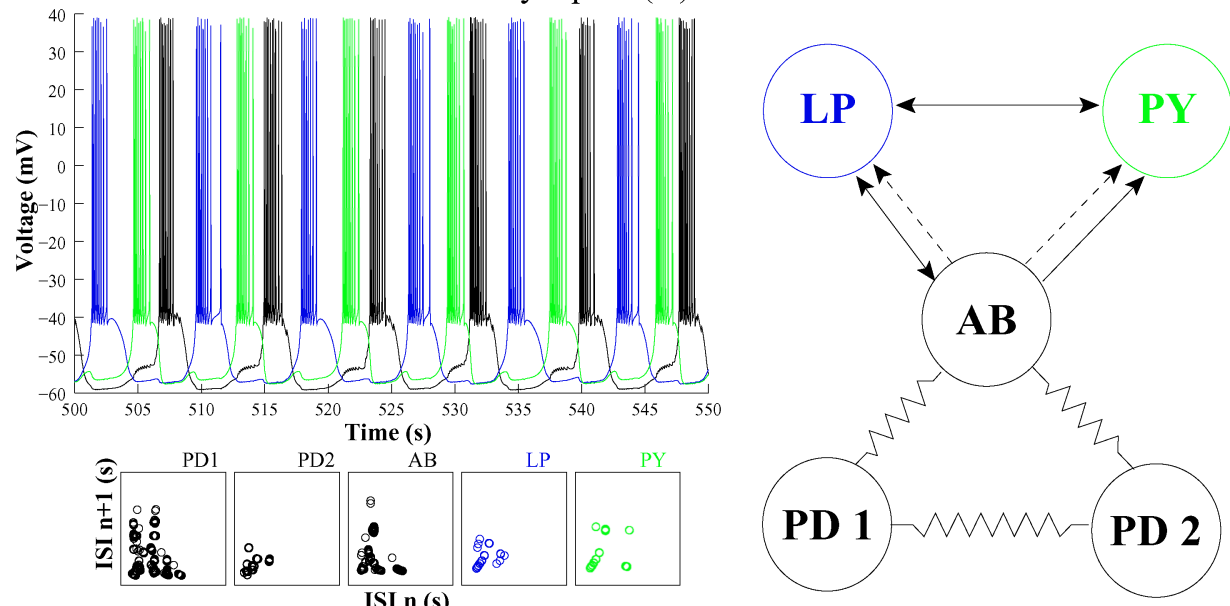

Circuit without Slow Chemical Synapses (B)
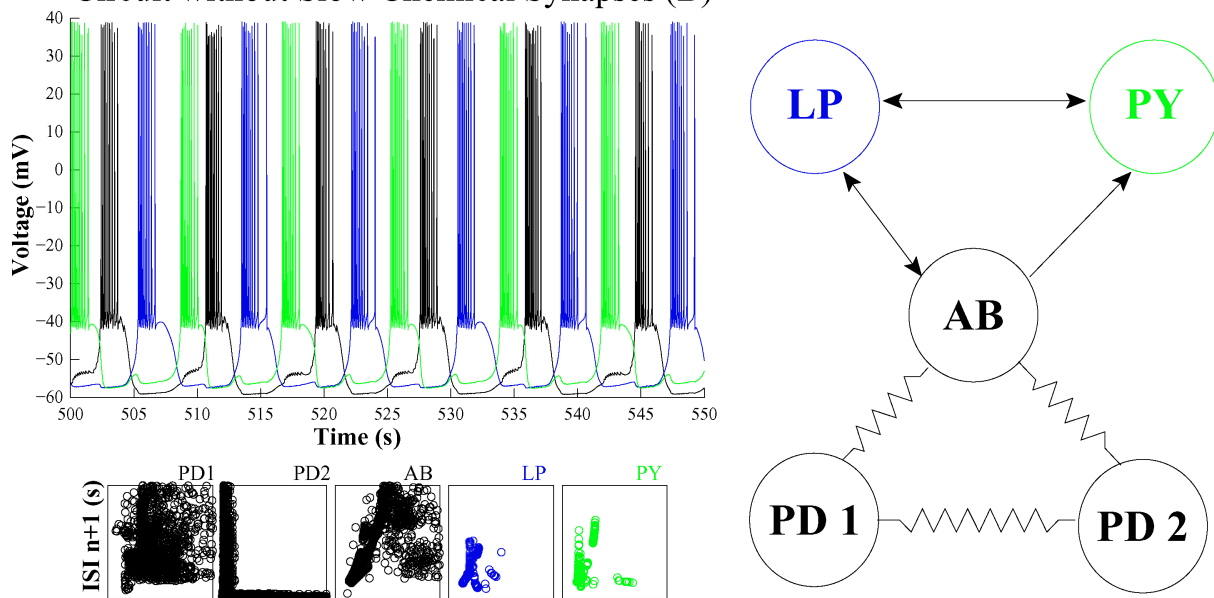

FIGURE 3. (Color online) Rhythms generated in the two circuits and neural signatures of each cell. Top panels: circuit with slow connections. Bottom panels: circuits without the slow connections. Axis length in all intraburst ISI return maps is $0.8 \mathrm{~s}$.

\section{Signatures are shaped by the network connections}

Before establishing the connections in the two circuits we have placed each neuron (except $\mathrm{AB}$ ) in a chaotic regime. The $\mathrm{AB}$ is tuned into a regular spiking-bursting behav- 
ior. We have chosen a chaotic spiking regime for the PY and one of the PD neurons, while the second PD and the LP are set into a chaotic spiking-bursting behavior (for details see $[13,14])$. When we connect the neurons in the circuits specified above, a characteristic triphasic rhythm arises independently of the mode of activity in isolation and the particular connection topology used in the network. The triphasic rhythm consists of an alternation of regular bursts in the activity of the AB, LP and PY neurons in this sequence (PDs follow the AB phase as mentioned before). Square panels in Fig. 3 show the interspike interval return plots for the different connection configurations.

Top square panels in Fig. 3 display the intraburst ISI return maps for the circuit with slow chemical synapses. Bottom square panels show the ISIs for the circuit without slow connections. The ISIs of network elements dramatically change from those corresponding to isolated neurons (cf. Fig. 1, note the different time scales in the axes). When the connections are established, the neuron signatures change even for the $\mathrm{AB}$ that also had a regular dynamics in isolation. In all simulations the networks display a regular triphasic rhythm. Regular in this context means that the slow wave duration remains constant from burst to burst. Each neuron in the network has a characteristic ISI signature. The signatures depend both on the particular settings of the individual neurons and on the topology of connections, but this topology is the final shaper of the neural signatures. The results reported in this section can be reproduced with other neuron models and network architectures [13, 14]. In general, the presence of slow chemical connections produced a more precise ISI return plot than those corresponding to the circuits without the slow synapses. PD1 and PD2 neurons were placed in two different modes in activation, and had major differences in the ISI plots in the circuit without slow connections. Simulations also show that in spite of its intrinsic regular bursting behavior, the $\mathrm{AB}$ can have complex signatures. Interestingly, LP and PY neurons had, in general, very similar and precise signatures in our simulations. These neurons are the only units that receive at least a double inhibition in the two circuits.

\section{MULTICODING BURSTS}

Another open question from the biology is related to the ability of a circuit to recognize a specific signature and, as a consequence, change its behavior. In this context, 'to recognize' a neural signature means that the reader system displays a different behavior when it receives different neural fingerprints, independently of the characteristics of the slow wave of the signal. It is unknown whether bursting neurons have cellular mechanisms to decode precise temporal information in the spike trains, besides those needed to detect changes in the period or in the phase relationships of input signals. This question is also difficult to address experimentally as it is hard to alter the ISI distribution without modifying the properties of the slow waves in vitro. Our goal in this section is to show, using model neurons and networks, that the spike distribution inside the bursts can be identified by an external system, i.e. another CPG. For this goal we will use a well known Liu et al. model of stomatogastric neurons, described in [22], to generate the characteristic spiking-bursting behavior of these cells. This model is a single compartment conductance-based description based on in vitro experiments on living cells [23]. The details of the model and the values of the parameters for each 
neuron in our simulations are described in [16].

\section{Signature emitter and reader CPGs}

Neural signatures arise within the particular rhythm generated by the CPG $[11,24$, 25]. Thus, we have built a network where the generation of different signatures, in an emitter $\mathrm{CPG}$, coexists with other functionality. A reader CPG will react to the signals produced in the emitter system. The network architecture of the emitter CPG is built with the idea to model a generic CPG composed of neurons $M, E_{1}, E_{2}$ and $I$ (see upper panel in Fig. 5). This CPG produces a well-defined rhythm while, at the same time, some of its neurons (in this case, $E_{1}$ and $E_{2}$ ) generate different signatures. We consider these later neurons signature emitter cells. To ensure that these neurons generate signals with the same burst duration and number of spikes, we use a pacemaker group $\left(M, E_{1}\right.$ and $E_{2}$ neurons) similar to the one present in the pyloric CPG [24]. The neurons of this group are electrically coupled by gap junctions (for details see [16]). Consequently, they fire together with the same slow wave frequency. However, as we will see later, the temporal distribution of spikes within the bursts is different because the individual behavior of each cell and the connections that they receive are not exactly the same (see [16]). In all cases considered, we want that this CPG generates a proper rhythm. This rhythm consists in the fire alternation of the pacemaker group and the $I$ neuron.

We will consider two reader CPGs that are also generic CPGs composed of neurons $R, N_{1}, N_{2}$ and $N_{3}$. The only difference between these two CPGs are the connectivity parameters among their neurons (see [16]). This difference will change the CPG reaction to the incoming signals: one of the reader CPGs will react to distinct neural signatures, while the other will react to distinct slow wave frequencies.

The emitter CPG neurons are connected to the $R$ cell of the reader CPGs through fast inhibitory graded chemical synapses. The $R$ neuron receives the signal from the signature emitter system and propagates it through the reader CPGs. We use different values for the synaptic conductances $\alpha$ and $\beta$ to control the presence of a signature at a given time (see top panel in Fig. 3). In all the simulations performed, one of the connections between the neurons of the emitter system and the reader CPGs is stronger than the other (with a higher conductance, in the order of $0.1 \mathrm{mS}$ vs $0.009 \mathrm{mS}$ ). Thus, the $R$ cell in each circuit receives the signature of one predominant emitter neuron at a time. In living systems, a similar effect can be produced by neuromodulators that are able to reconfigure the neuronal networks [26, 27, 28, 29, 30, 31]. The $N_{1}, N_{2}$ and $N_{3}$ neurons produce the CPG rhythm. The $R$ neuron receives a feedback from the rest of the CPG through the $N_{1}$ and $N_{3}$ cells.

Thus, our final circuit consists of three CPGs, each one performing a different task (generating different rhythms) but intercommunicated with signals that can have different signatures and slow wave frequencies. The emitter CPG drives the behavior of the reader CPGs as their specific rhythm depends on the signals received from the emitter. 


\section{Emitter signals}

The emitter CPG can produce signals with different slow wave rhythms and also different signatures. In particular, we will consider four different signals within the collective rhythm generated by the signature emitter CPG: two different slow waves, F1' (with a burst frequency of $1.38 \mathrm{~Hz}$ ) and F2' (with a burst frequency of $1.49 \mathrm{~Hz}$ ), and two different signatures on top of each of them (see Fig. 4). The parameters used in these simulations are described in [16]. The signals have the same burst duration and the same number of spikes per burst ( 6 spikes). When these signals are read by the reader CPGs, their response depends on the particular spike distribution inside the burst of each input signal or the specific frequency of the rhythm.
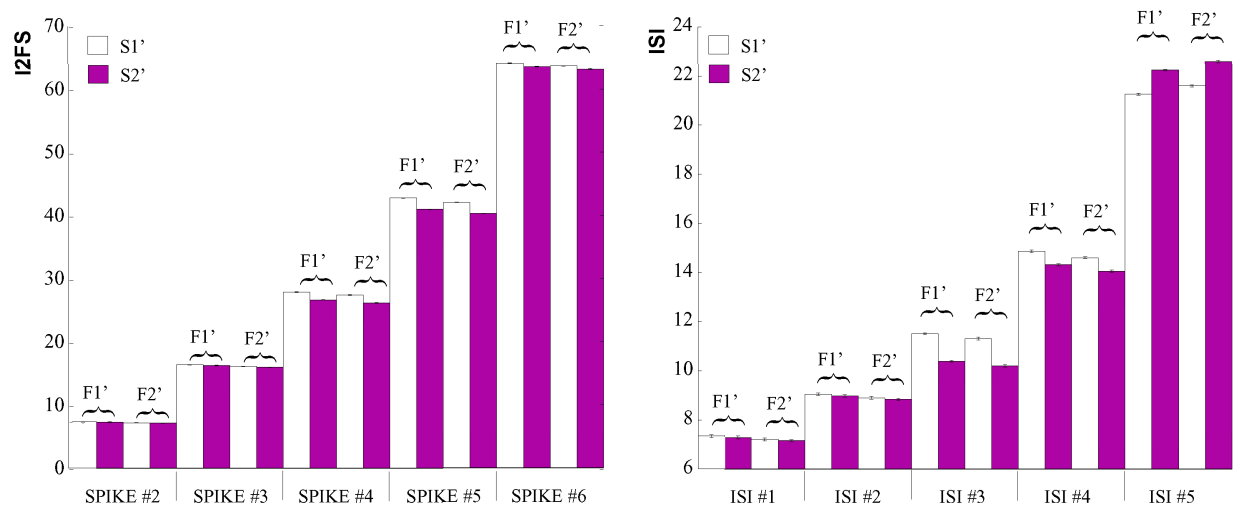

FIGURE 4. (Color online) Signatures of the emitter CPG. Left panel: Bar plot of the mean time interval from each action potential to the first spike (I2FS) for the different bursting signals generated by the signature emitter CPG. Right panel: Bar plot of the average ISI distribution for the same signals. For each spike, the first two bars correspond to signals with frequency F1' and the last two bars correspond to signals with frequency F2'. A particular signature Sx' can be on top of the rhythms with these two frequencies. Thus, we can say that the emitter CPG can also produce two different signatures (S1' and S2') on signals with two different slow wave rhythms (F1' and F2'). The labels 1 and 2 in these signals correspond to neurons E1 and E2, respectively, of the emitter CPG. Units are ms.

\section{Distinct readers react to different features of the signals}

To assess the effect of multiple coding in the input signals, we observe the reaction of the reader CPGs to the different signals produced by the emitter CPG. The $E$ neurons of the emitter system can thus generate signals with two different slow waves frequencies, each of them with two different signatures. These signals are received in the $R$ cell and then propagated to other neurons in the reader CPGs.

The signature reader CPG (Fig. 5, left panels) reacts to the distinct signatures of the incoming signals, independently of the emitter slow wave frequencies. The response of $R$ to signals with signature $\mathrm{S} 1$ ' contributes to the generation of a biphasic rhythm, with firing sequence $N_{1}-N_{3}$ and $N_{2}$ in silent. However, with signature S2', the reader CPG generates a triphasic rhythm, with firing sequence $N_{1}-N_{2}-N_{3}$. Rhythms generated when 

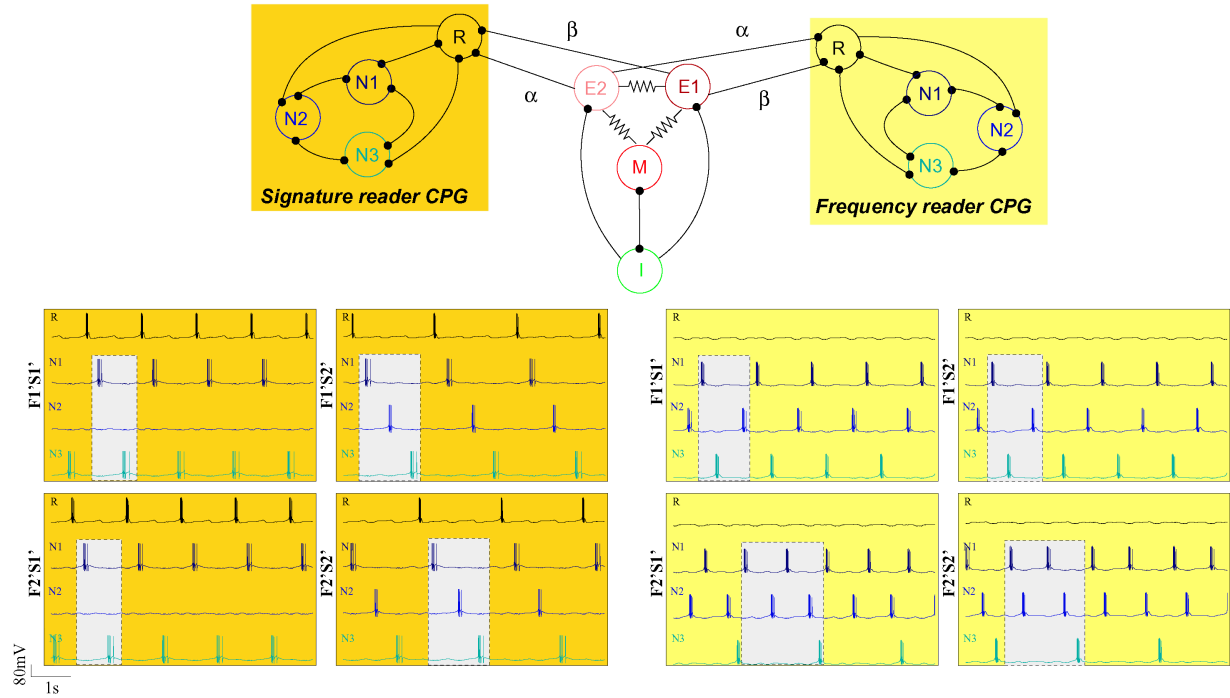

FIGURE 5. (Color online) Distinct reaction of the different CPG readers to four different signals in the emitter CPG. Left panels: reaction of the signature reader CPG. Right panels: reaction of the frequency reader CPG.

$R$ receives signature $\mathrm{S} 1$ ' have a higher slow wave frequency than those generated with S2'.

In the frequency reader CPG (Fig. 5, right panels) the rhythm generated does not depend on the signature. In this case the CPG generates different rhythms as a function of the frequency of the signal received. With F1' (top panels) the CPG generates a triphasic rhythm that consists in the firing alternation of $N_{1}, N_{3}$ and $N_{2}$ neurons, in this order. With F2' (bottom panels) the CPG generates a rhythm with firing sequence $N_{1}-N_{2}-N_{1}-N_{2}-N_{3}$. Thus, the two circuits use different features of the signal to produce distinct rhythms.

\section{DISCUSSION}

What subcellular mechanism can be responsible for the distinct responses of a neural reader to different interspike interval distributions? There is not a simple answer to this question. Obviously, the synaptic currents from the emitters are the origin for the different behaviors displayed by the signature reader CPG. The integration of these currents with the ongoing intracelullar dynamics of the reader neurons determines the characteristics of the response. The interaction of slow dynamics such as that of the calcium concentration with the fast kinetics of most ionic channels [32] can allow a distinct integration for incoming signals with a particular spike distribution [16]. Models have shown that the participation of calcium in the neuronal dynamics can regulate the timing precision of action potentials in specific regions of the burst [33]. The different resonant properties of intracellular dynamics have been suggested as the 
minimal dynamical mechanism to identify non-trivial sequences of spike timings [34].

Many neural systems (vertebrate and invertebrate) display very precise timings in the spiking activity of different neurons, e.g. see [35, 36, 37, 38]. Although we have restricted our study to spiking-bursting CPG models, neural signatures can be a general mechanism present in other neural networks to implement preferred input/output relations. These preferred input/output relations can explain why there are many silent neurons in spite of the large number of incoming signals that they receive in a few milliseconds. The existence of cellular mechanisms to identify the origin of individual neural signals, and the study of information processing based on this identification have been neglected in the context of theoretical approaches to the nervous system. Neurons could not only discriminate specific interspike frequencies $[5,6]$, but also react selectively to input from specific cells identified by their signature [16].

Other bursting neurons show the presence of regions of focalized timing precision in the middle of the bursts [39]. In these neurons, the more variable first spikes could encode information about the duration and/or number of spikes in the burst [39]. Information processing based on the identification of spike timings in bursting neurons can be a powerful strategy for neural systems that greatly enhances the capacity of these networks.

\section{ACKNOWLEDGMENTS}

This work was supported by Fundación BBVA, MEC BFU2006-07902/BFI and MEC TIN2004-04363-C03-03.

\section{REFERENCES}

1. D. A. McCormick, and D. Contreras, Annu. Rev. Physiol. 63, 815-846 (2001).

2. J. E. Lisman, Trends Neurosci. 20, 38-43 (1997).

3. F. G. Pike, R. M. Meredith, A. W. A. Olding, and O. Paulsen, J. Physiol. (Lond) 518, 571-576 (1999).

4. A. Kepecs, and J. Lisman, Network 14, 103-118 (2003).

5. E. Izhikevich, N. S. Desai, E. C. Walcott, and F. C. Hoppensteadt, Trends Neurosci. 26(3), 161-167 (2003).

6. R. Krahe, and F. Gabbiani, Nature Rev. Neurosci. 5, 13-24 (2004).

7. A. M. Oswald, M. J. Chacron, B. Dorion, J. Bastian, and L. Maler, J. Neurosci. 24, 4351-4362 (2004).

8. R. C. Elson, R. Huerta, H. D. I. Abarbanel, M. I. Rabinovich, and A. I. Selverston, J. Neurophysiol. 82, 115-122 (1999).

9. P. Varona, J. J. Torres, R. Huerta, H. D. I. Abarbanel, and M. I. Rabinovich, Neural Networks 14, $865-875$ (2001).

10. P. Varona, J. J. Torres, H. D. I. Abarbanel, M. I. Rabinovich, and R. Elson, Biol. Cybern. 84, 91-101 (2001).

11. A. Szücs, R. D. Pinto, M. I. Rabinovich, H. D. I. Abarbanel, and A. I. Selverston, J. Neurophysiol. 89, 1363-1377 (2003).

12. A. Szücs, H. D. I. Abarbanel, M. I. Rabinovich, and A. I. Selverston, European J. Neurosci. 2 , 763-772 (2005).

13. R. Latorre, F. B. Rodríguez, and P. Varona, Lect. Notes Comput. Sc. 2415, 160-166 (2002).

14. F. B. Rodríguez, R. Latorre, and P. Varona, Lect. Notes Comput. Sc. 2415, 167-173 (2002).

15. R. Latorre, F. B. Rodríguez, and P. Varona, Neurocomputing 58-60, 535-540 (2004).

16. R. Latorre, F. B. Rodríguez, and P. Varona, Biol. Cybern. 95, 169-183 (2006). 
17. R. Latorre, F. B. Rodríguez, and P. Varona, Neurocomputing, In press (2007).

18. A. O. Komendantov, and N. I. Kononenko, J. Theor. Biol. 183, 219-230 (1996).

19. A. J. Dekhuijzen, and J. Bagust, J. Neurosci. Methods 67, 141-147 (1996).

20. J. P. Segundo, G. Sugihara, P. Dixon, M. Stiber, and L. F. Bersier, Neuroscience 87, 741-766 (1998).

21. M. A. Fitzurka, and D. C. Tam, Biol. Cybern. 80, 309-326 (1999).

22. A. Liu, J. Golowasch, E. Marder, and F. Abbott, J. Neurosci. 18, 2309-2320 (1998).

23. G. G. Turrigiano, G. LeMasson, and E. Marder, J. Neurosci. 15, 3640-3652 (1995).

24. A. I. Selverston, and M. Moulins, editors, The Crustacean Stomatogastric System: a Model for the Study of Central Nervous System., Springer-Verlag, Berlin Heidelberg New York London Paris Tokyo, 1987.

25. R. M. Harris-Warrick, E. Marder, A. I. Selverston, and M. Moulins, editors, Dynamic Biological Networks: The Stomatogastric Nervous System., Cambridge, MA: MIT Press, 1992.

26. E. Marder, and R. L. Calabrese, Physiol. Rev. 76, 687-717 (1996).

27. E. Marder, and D. Bucher, Curr. Biol. 11, R986-R996 (2001).

28. M. P. Nusbaum, D. M. Blitz, A. M. Swense, D. Wood, and E. Marder, Trends Neurosci. 24, 146-154 (2001).

29. M. P. Nusbaum, and M. P. Beenhakken, Nature 417, 343-350 (2002).

30. M. A. Masino, and R. L. Calabrese, J. Neurosci. 22, 4418-4427 (2002).

31. M. A. Masino, and R. L. Calabrese, J. Neurophysiol. 87, 1603-1615 (2002).

32. S. Ramaswamy, F. Baroni, P. Varona, and G. G. de Polavieja, Neurocomputing, In press (2007).

33. F. Baroni, J. Torres, and P. Varona, Lect. Notes Comput. Sci. 3561, 106-115 (2005).

34. F. Baroni, and P. Varona, Neurocomputing, In press (2007).

35. P. Reinagel, and R. C. Reid, J. Neurosci. 20, 5392-5400 (2000).

36. Z. Chi, and D. Margoliash, Neuron 32, 899-910 (2001).

37. P. Reinagel, and R. C. Reid, J. Neurosci. 22(16), 6837-6841 (2002).

38. J. D. Hunter, and J. G. Milton, J. Neurophysiol. 90, 387-394 (2003).

39. D. Campos, C. Aguirre, E. Serrano, F. B. Rodríguez, G. de Polavieja, and P. Varona, Neurocomputing, In press (2007). 
Copyright of AIP Conference Proceedings is the property of American Institute of Physics and its content may not be copied or emailed to multiple sites or posted to a listserv without the copyright holder's express written permission. However, users may print, download, or email articles for individual use. 